\title{
Long-term efficacy of a-lipoic acid on cardiovascular autonomic function in type 2 diabetes
}

\author{
Rumyana Dimova, Tsvetalina Tankova, Nevena Chakarova, Greta Grozeva, Lilia Dakovska \\ Department of Diabetology, Clinical Centre of Endocrinology, Medical University Sofia, Bulgaria
}

Background: Although a-lipoic acid is the only agent for pathophysiological treatment of diabetic neuropathy, the available randomized clinical studies provide no evidence on the long-term benefits of this therapy.

Aim: The present study aimed to evaluate the long-term efficacy of a-lipoic acid on cardiovascular autonomic function in type 2 diabetes (T2D).

Material: A total of 94 subjects (46 males) with T2D, of mean age $49.4 \pm 14.1$ years, mean BMI $27.5 \pm 7.6 \mathrm{~kg} / \mathrm{m} 2$, were included in a cross-sectional study. The participants were divided into two groups according to treatment - treated with a-lipoic acid for a period of five years and treatmentnaïve.

\begin{tabular}{lccc}
\hline & a-lipoic acid- & a-lipoic acid & $p$ \\
\hline number & 53 & 41 & \\
sex (male/female) & $28 / 25$ & $18 / 23$ & \\
age (years) & $51.8 \pm 12.8$ & $42.6 \pm 15.6$ & 0.003 \\
BMI (kg/m $)$ & $28.7 \pm 8.1$ & $24.0 \pm 4.8$ & 0.002 \\
HbAlc $(\%)$ & $8.5 \pm 2.0$ & $9.5 \pm 2.0$ & 0.015 \\
diabetes duration & $10.5 \pm 7.8$ & $9.4 \pm 6.1$ & $<0.001$ \\
(years) & & & \\
\hline Data are means \pm standard deviation & & \\
\hline
\end{tabular}

\section{Methods:}

- HbA1c was measured in whole blood immunoturbidimetrically.

- Weight and height were measured and BMI was calculated.

- Cardiovascular autonomic function was evaluated by autonomic nervous system (ANS) monitoring technology ANSAR ANX 3.0 based on spectral analysis of HRV with concurrent spectral analysis of respiratory activity, measuring the balance between both ANS branches using the following clinical tests: 1) deep breathing (E/I ratio), 2) Valsalva maneuver, and 3) standing from a seated position (30:15 ratio) tests.

- Statistical analysis - SPSS 20.0 for Windows (SPSS, Chicago, USA).

Results:

Confirmed cardiovascular autonomic dysfunction in the groups according to the

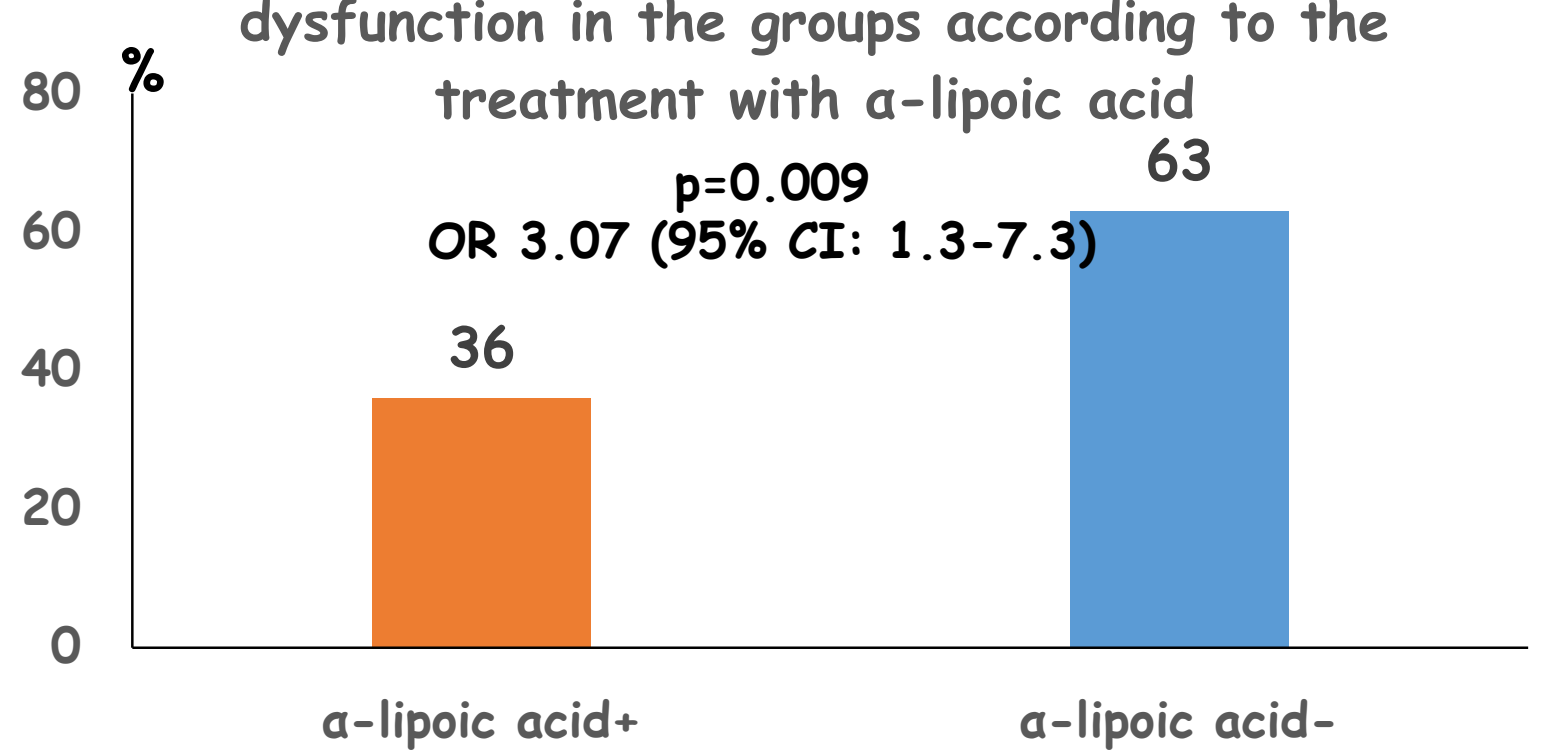

Postural hypotension in the groups according

to the treatment with a-lipoic acid

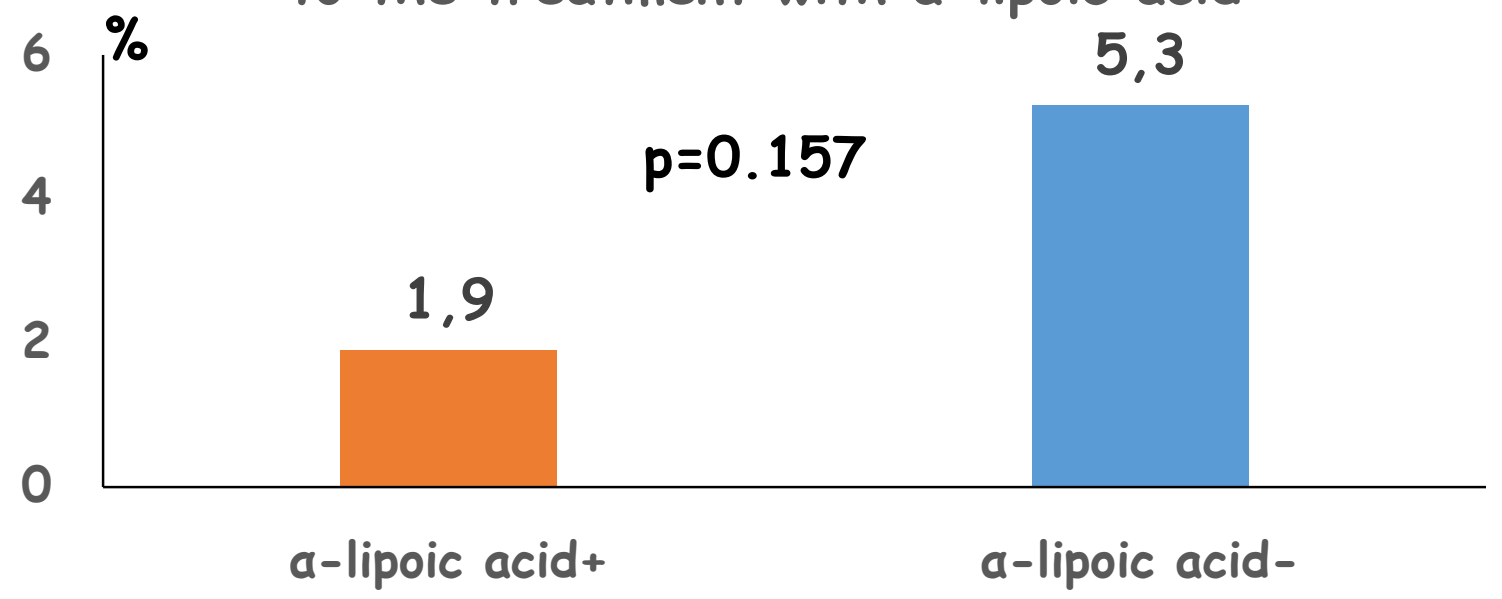

Abnormal sympathetic (LFa) and parasympathetic (RFa) activity at rest and during standard clinical tests in \% in the groups according to the treatment with a-lipoic acid.
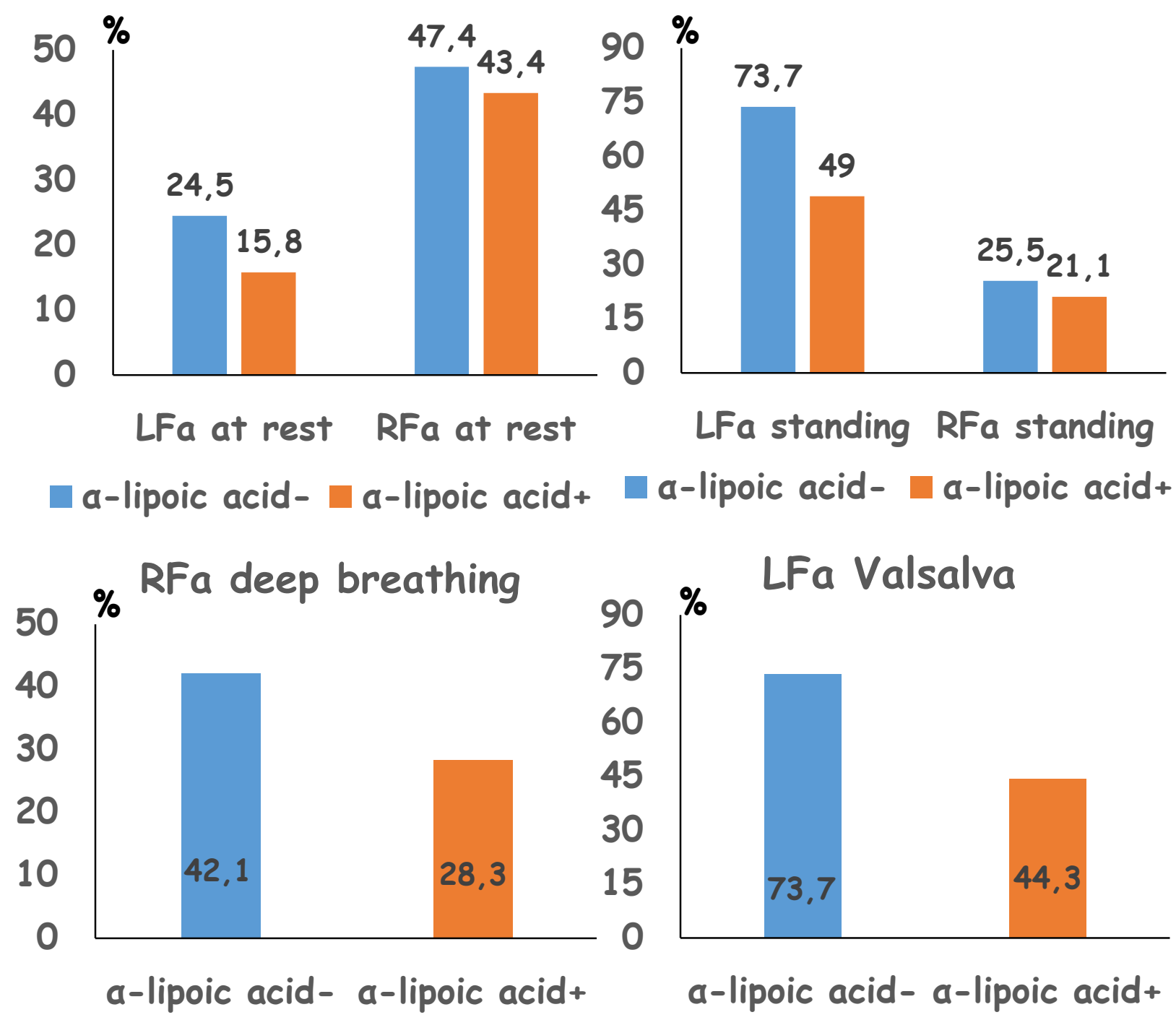

Percentage of pathological autonomic tests and the number of pathological tests per subject in the groups according to the treatment with a-lipoic acid
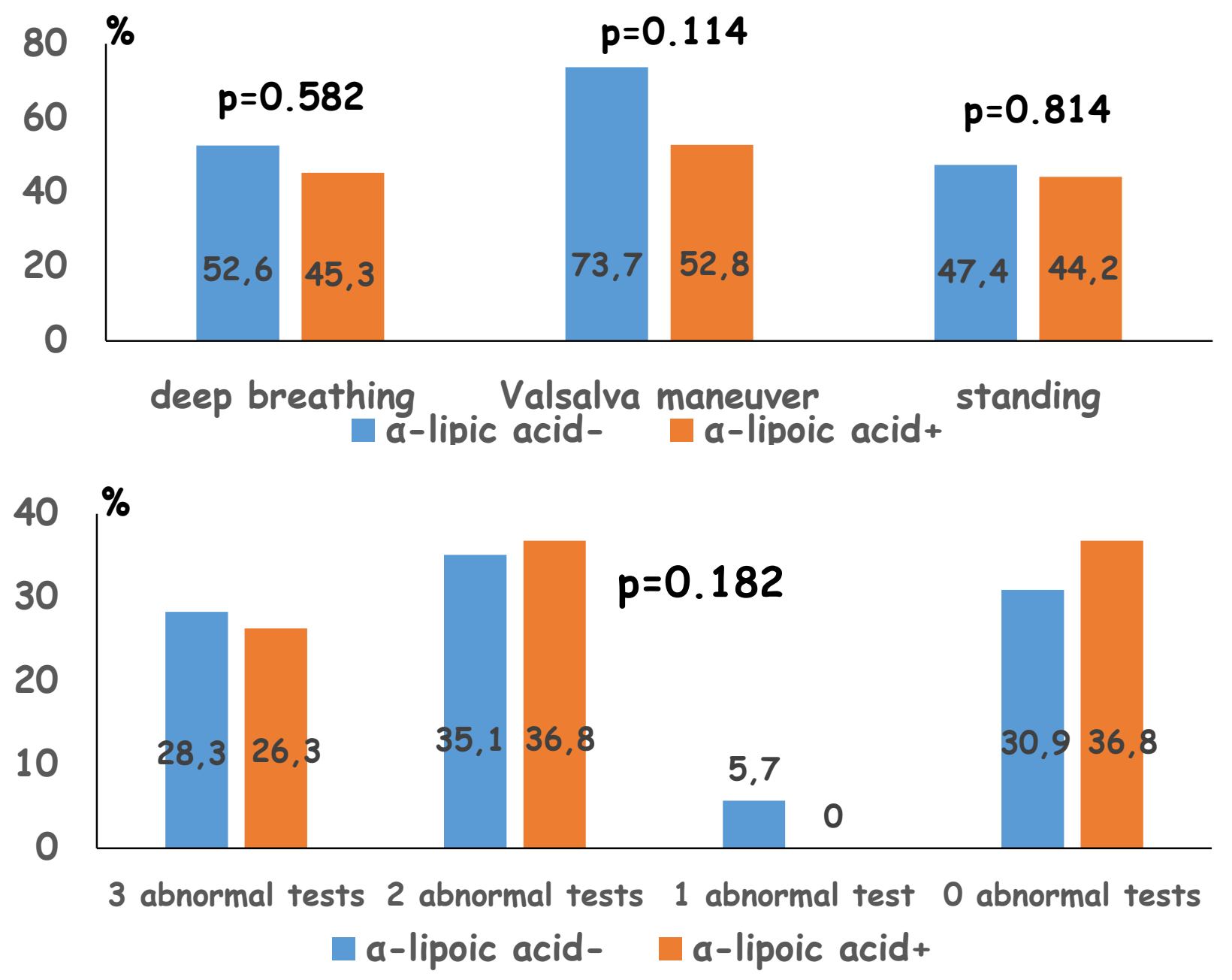

Discussion: The results of the present study have confirmed the long-term beneficial effect of a-lipoic acid on cardiovascular autonomic function in subjects with T2D, independently of age, diabetes duration and obesity.

This poster does not contain any trade names and does not cover any unapproved uses of specific drugs, other products or devices. There are no conflicts of interest.

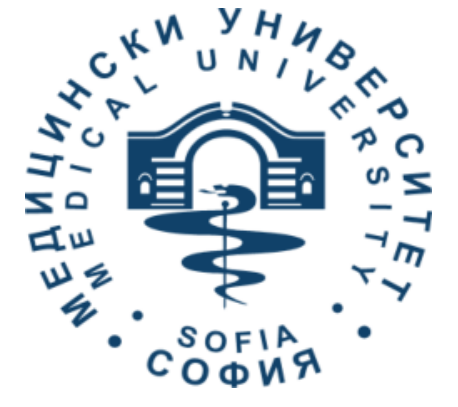

